\title{
Effects of coronary revascularisation on myocardial blood flow and coronary vasodilator reserve in hibernating myocardium
}

\author{
D Pagano, F Fath-Ordoubadi, K J Beatt, J N Townend, R S Bonser, P G Camici
}

\begin{abstract}
Objective-Previous studies have suggested that resting myocardial blood flow is within normal limits in most chronically dysfunctional left ventricular segments which improve function after coronary artery revascularisation (hibernating myocardium). The aim of this study was to assess myocardial blood flow and coronary vasodilator reserve in hibernating myocardium before and after coronary revascularisation.

Patients and methods - 30 patients with multivessel coronary disease undergoing coronary revascularisation (21 patients with bypass grafting and nine with coronary angioplasty), and 21 age and sex matched healthy volunteers (controls). Myocardial blood flow ( $\mathrm{MBF}, \mathrm{ml} / \mathrm{min} / \mathrm{g}$ ) was measured by positron emission tomography using oxygen-15 water at rest and after dipyridamole (MBFdip, $0.56 \mathrm{mg} / \mathrm{kg}$ in four minutes). Coronary vasodilator reserve was calculated as MBFdip/ MBF. Regional wall motion was assessed with echocardiography.

Results-Before revascularisation there were 48 remote and 275 dysfunctional myocardial segments, of which 163 (59\%) improved function after revascularisation (hibernating). In hibernating segments coronary vasodilator reserve before revascularisation was significantly lower than in remote segments $(1.97(0.7), \mathrm{p}<0.0001)$ and controls $(3.2(1.5), \mathrm{p}<0.0001)$. In hibernating segments, myocardial blood flow remained unchanged after revascularisation $(0.94(0.3) v 0.95$ $(0.3) \mathrm{ml} / \mathrm{min} / \mathrm{g}, \mathrm{p}=0.3)$ while coronary vasodilator reserve increased $(1.47(0.7) v 1.98(1.0)$, $\mathrm{p}<0.0001)$. Myocardial blood flow was similar in remote, hibernating segments before and after revascularisation and in controls.

Conclusions-This study confirms that myocardial blood flow at rest in hibernating myocardium is within normal limits in most segments, and that hibernating myocardium is characterised by an impaired coronary vasodilator reserve which improves significantly after coronary revascularisation.
\end{abstract}

(Heart 2001;85:208-212)

Keywords: hibernating myocardium; myocardial blood flow; heart failure; positron emission tomography

Cardiothoracic Surgical Unit, Queen Elizabeth Hospital, Birmingham, UK D Pagano

R S Bonser

University Department of Cardiology, Queen Elizabeth Hospital, Birmingham

J N Townend

MRC Clinical Sciences Centre and National

Heart and Lung

Institute, Imperial

College School of

Medicine,

Hammersmith

Hospital, Du Cane

Road, London W12

ONN, UK

F Fath-Ordoubadi

K J Beatt

P G Camici

Correspondence to: Professor Camici

paolo.camici@csc.mrc.ac.uk

Accepted 2 October 2000
Chronic post-ischaemic left ventricular dysfunction in myocardium subtended by a stenotic coronary artery can be improved by revascularisation. This phenomenon of reversible dysfunction has been termed "myocardial hibernation". ${ }^{12}$ It has been hypothesised that hibernation is an "adaptation" to chronic reduction in resting myocardial blood flow secondary to coronary artery stenosis. ${ }^{2}$ However, this is contentious as many studies have suggested that basal myocardial blood flow is not significantly reduced even in the presence of severe coronary artery stenoses, ${ }^{3}$ while there is a progressive reduction of coronary vasodilator reserve with increasing stenosis severity. ${ }^{3}$ It has also been demonstrated that, in the majority of cases, baseline myocardial perfusion in chronically dysfunctional myocardium which recovers function after revascularisation is similar to that of myocardium with normal contractile function in the same patients ${ }^{4}$ and to that of normal healthy subjects. Furthermore, non-infarcted, collateral dependent dysfunctional myocardium has normal resting myocardial perfusion but a reduced coronary vasodilator reserve compared to ischaemic but normally functioning segments. ${ }^{5}$

Our study aimed to assess non-invasively regional myocardial blood flow and coronary vasodilator reserve in hibernating myocardium before and after coronary revascularisation using positron emission tomography (PET), and to compare them with regions with normal contractile function subtended by a normal coronary artery (remote regions) and myocardium of healthy subjects.

\section{Methods}

STUDY POPULATION

The patient population consisted of 30 subjects (28 males; mean (SD) age 56 (10) years) with coronary artery disease ( $>6$ months) undergoing coronary artery bypass grafting (CABG, 21 patients) or percutaneous transluminal coronary angioplasty (PTCA, nine patients). All patients were in sinus rhythm and had suffered at least one (range 1-3) Q wave myocardial infarction 30 (14) months before the study. Seven patients were diabetic and five were hypertensive. The left ventricular ejection fraction (LVEF) was 30 (11)\%. Patients were receiving treatment with angiotensin converting enzyme (ACE) inhibitors (20 patients), diuretics (15 patients), digoxin (5 patients), nitrates (10 patients), calcium channel blockers (13 patients), and $\beta$ blockers (9 patients). All medical treatment was withdrawn at least 24 hours before the study day both at baseline and follow 
Table 1 Myocardial blood flow and coronary vasodilator reserve in hibernating segments

\begin{tabular}{lllc}
\hline & Pre-revascularisation & Post-revascularisation & p Value \\
\hline $\mathrm{MBF}(\mathrm{ml} / \mathrm{min} / \mathrm{g})$ & $0.92(0.30)$ & $0.95(0.32)$ & 0.29 \\
$\mathrm{MBF}-\operatorname{corr}(\mathrm{ml} / \mathrm{min} / \mathrm{g})$ & $0.94(0.34)$ & $0.95(0.27)$ & 0.88 \\
$\mathrm{MBF}-d i p(\mathrm{ml} / \mathrm{min} / \mathrm{g})$ & $1.31(0.65)$ & $1.86(1.0)$ & $<0.0001$ \\
$\mathrm{CVR}$ & $1.48(0.66)$ & $2.0(1.0)$ & $<0.0001$ \\
CVR-corr & $1.47(0.66)$ & $1.98(1.0)$ & $<0.0001$ \\
\hline
\end{tabular}

All values are mean (SD).

$\mathrm{MBF}$, resting myocardial blood flow ( $\mathrm{ml} / \mathrm{g} / \mathrm{min}) ; \mathrm{MBF}-\mathrm{dip}$, myocardial blood flow after dipyrida$\mathrm{mole}(\mathrm{ml} / \mathrm{g} / \mathrm{min})$; CVR, coronary vasodilator reserve; MBFcorr, corrected for rate-pressure product; CVRcorr, corrected for rate-pressure product.

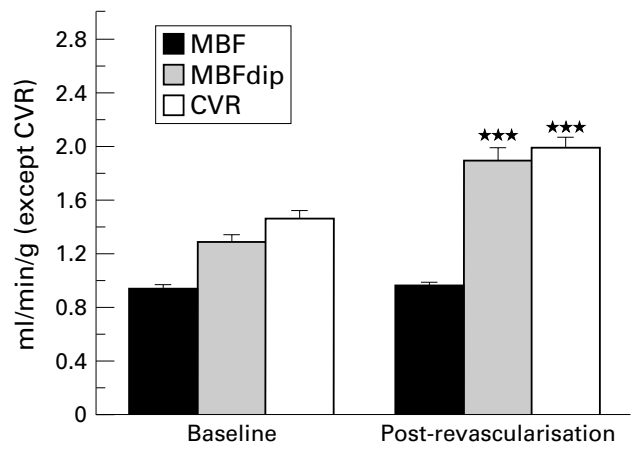

Figure 1 Perfusion data on 163 hibernating myocardial segments before (baseline) and after coronary revascularisation. $M B F$, myocardial blood flow; $M B F d i p$, post-dipyridamole myocardial blood flow; CVR, coronary vasodilator reserve. ${ }^{\star \star *} p<0.0001$.

up. Patients were asked to abstain from drinks containing caffeine for at least 24 hours before the study. Partial data on 19 patients has been reported previously. ${ }^{6}$

A group of 21 age (53(12) years) and sex matched normal volunteers ${ }^{6}$ served as controls for the myocardial blood flow and coronary vasodilator reserve measurements. All controls had no history of cardiac disease, a low risk profile, normal physical examination, normal resting ECGs, and negative exercise tests in response to high workloads.

The study was approved by the local ethics committee and written informed consent was obtained by all the patients before the study. The radiation exposure was licensed by the UK administration of radioactive substances advisory committee (ARSAC).

CORONARY ARTERIOGRAPHY

Selective right and left coronary arteriography in multiple views was performed with the Judkins technique. Coronary arteriography was repeated in the nine patients undergoing PTCA three months after the procedure.

RADIONUCLIDE VENTRICULOGRAPHY

LVEF was calculated by radionuclide ventriculography using standard techniques. ${ }^{7}$ LVEF was reassessed three months following PTCA and six months following CABG. The analysis

Table 2 Myocardial blood flow and coronary vasodilator reserve in remote segments

\begin{tabular}{llll}
\hline & Pre-revascularisation & Post-revascularisation & p Value \\
\hline $\mathrm{MBF}(\mathrm{ml} / \mathrm{min} / \mathrm{g})$ & $0.91(0.20)$ & $0.86(0.25)$ & 0.29 \\
$\mathrm{MBF}-\mathrm{corr}(\mathrm{ml} / \mathrm{min} / \mathrm{g})$ & $0.96(0.28)$ & $0.89(0.36)$ & 0.15 \\
$\mathrm{MBF}-\operatorname{dip}(\mathrm{ml} / \mathrm{min} / \mathrm{g})$ & $1.89(0.84)$ & $1.84(0.80)$ & 0.68 \\
$\mathrm{CVR}$ & $2.1(1.0)$ & $2.2(1.0)$ & 0.66 \\
CVR-corr & $1.97(0.70)$ & $2.2(1.2)$ & 0.10 \\
\hline
\end{tabular}

All values are mean (SD).

See table 1 for key to abbreviations. was performed by two experienced physicians blinded to all clinical details. The interobserver and intra-observer agreement was assessed $^{8}$ in a random subset of 10 patients. The mean of the differences in LVEF was 0.05 (1.5) $\%$ points and the $95 \%$ limits of agreement were $2.9-3 \%$.

TRANSTHORACIC ECHOCARDIOGRAPHY

Standard views ${ }^{9}$ were acquired. Resting wall motion/thickening was reassessed three months following PTCA (9 patients) and six months following CABG (21 patients). All the studies were performed by an experienced echocardiographer blinded to all the clinical details of the patients. Images were stored on conventional VHS tapes and analysed off-line by continuous display by two experienced observers who were blinded to the clinical, angiographic, and previous echocardiographic results of the patients. For analysis of wall motion, the left ventricle was divided in 16 segments. Regional wall motion/thickening was assessed for each segment and systolic wall motion was graded using a semiquantitative scoring system (American Society of Echocardiography $\left.{ }^{9}\right): 1=$ normal; $2=$ hypokinetic; 3 = akinetic; $4=$ dyskinetic. Dysfunctional segments improving wall motion at least one point at the follow up study were considered hibernating. In a random subset of 10 patients the inter-observer and the intra-observer $\kappa$ agreement was assessed: inter-observer $\kappa=0.82(95 \%$ confidence interval (CI) 0.69 to $0.95)$; intra-observer $\kappa=0.90$ (95\% CI 0.80 to $1.00)$.

PET MEASUREMENTS OF MYOCARDIAL BLOOD FLOW AND CORONARY VASODILATOR RESERVE The PET studies were performed in the patients before revascularisation, three months after PTCA or six months after CABG, and in the control subjects using an ECAT 931-08/12 scanner (CTI Inc, Knoxville, Tennessee, USA). ${ }^{10}$ Myocardial blood flow (MBF, ml/ $\mathrm{min} / \mathrm{g}$ ) was measured using $\mathrm{H}_{2}{ }^{15} \mathrm{O}$ as previously reported. ${ }^{11}$ Measurements were made at rest and two minutes after intravenous administration of dipyridamole $(0.56 \mathrm{mg} / \mathrm{kg}$ over four minutes, MBFdip). ${ }^{12}$ Coronary vasodilator reserve was calculated as the ratio of postdipyridamole myocardial blood flow to myocardial blood flow at baseline (MBFdip/ $\mathrm{MBF}){ }^{12}$ Because baseline myocardial blood flow is closely related to the rate-pressure product (RPP), ${ }^{13}$ an index of myocardial oxygen consumption, basal flow data were also corrected for the RPP using the following equation: $\mathrm{MBF}$ corr $=\mathrm{MBF} \times($ mean patient RPP/individual RPP). ${ }^{11}$

Reconstruction and analysis of the PET images was performed as previously described. ${ }^{11}$ Images were re-sliced in the short axis view and the left ventricle was divided into 16 segments comparable to the echo images, as previously described. ${ }^{14}$

STATISTICAL ANALYSIS

Data are expressed as mean (1 SD). Paired data were compared with the paired $t$ test. 


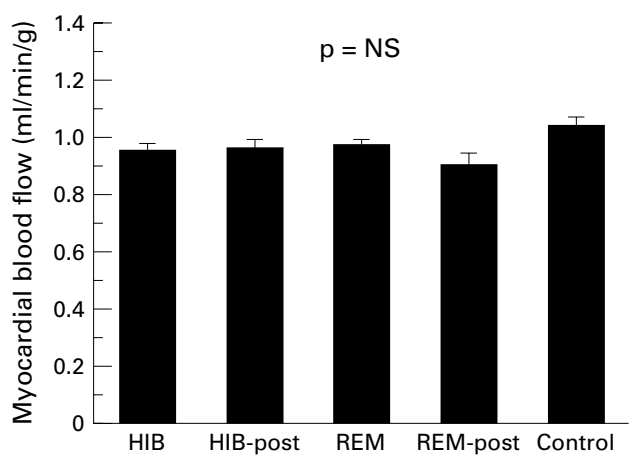

Figure 2 Comparison of myocardial blood flow between hibernating, remote (before and after revascularisation), and control myocardial segments. HIB, hibernation segments before revascularisation; REM, remote segments; -post refers to post-revascularisation.

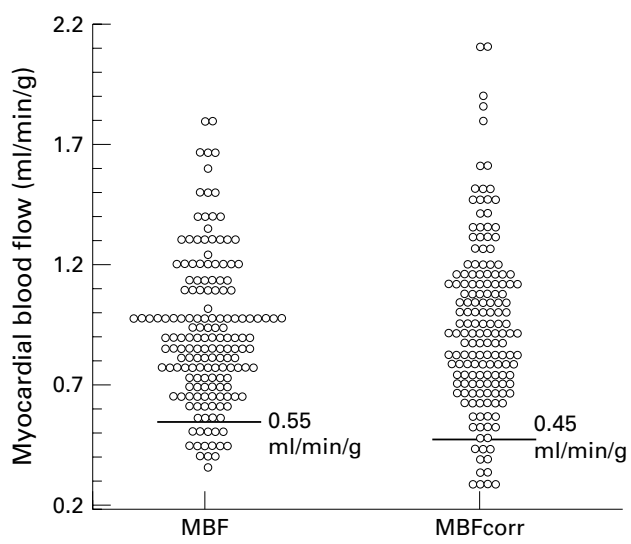

Figure 3 Scatterplot showing the distribution of myocardial blood flow (uncorrected and corrected for the rate-pressure product) before revascularisation in the hibernating segments. The thresholds of 0.55 and 0.45 $\mathrm{ml} / \mathrm{min} / \mathrm{g}$ represent the mean value minus 2 SDs of myocardial blood flow in control segments (uncorrected and corrected for the rate-pressure product). MBF, myocardial blood flow uncorrected for the rate-pressure product; $M B F$ corr, myocardial blood flow corrected for the rate-pressure product.

Simultaneous comparison of more than two sets of data was made using one way analysis of variance, followed by the Tukey-Kramer test to identify the source of variation. Significance was assumed for a probability value of $\mathrm{p}<0.05$ (two tailed).

\section{Results}

LEFT VENTRICULAR FUNCTIONAL OUTCOME

A total of 431 left ventricle segments were assessed by PET and echocardiography. Of these, 48 manifested normal contractile function and were subtended by a normal coronary artery (remote regions) and 383 segments were subtended by a stenotic $(>70 \%)$ coronary artery. Of these 383 segments, 370 were revascularised. Nine segments were excluded because of restenosis of the subtending vessel three months after PTCA. Of the remaining 361 revascularised segments, 275 were dysfunctional and 86 had normal baseline wall motion. At follow up 163/275 (59\%) of dysfunctional segments improved contractile function (hibernating segments) while dysfunction in the remaining 112 segments remained unchanged. The global LVEF increased from $30 \quad(11) \%$ to $37 \quad(10) \%$

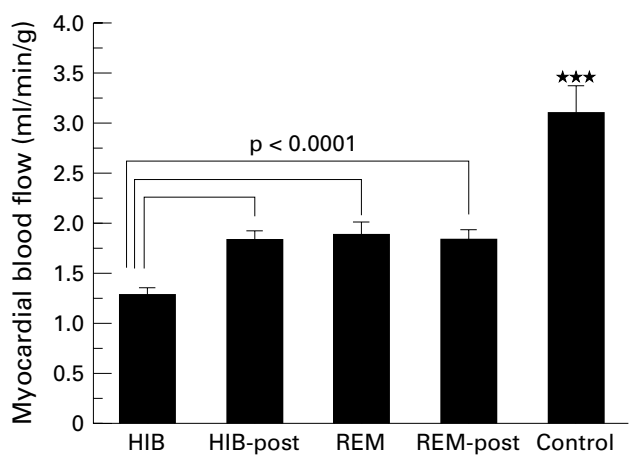

Figure 4 Comparison of post-dipyridamole myocardial blood flow between hibernating, remote (before and after revascularisation), and control myocardial segments. See fig 2 for key to abbreviations.

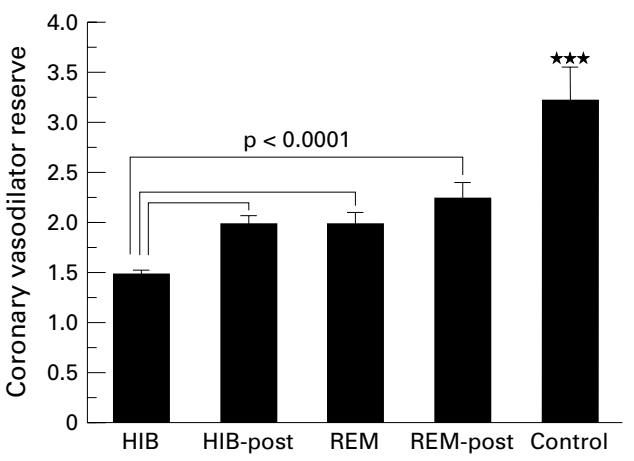

Figure 5 Comparison of coronary vasodilator reserve between hibernating, remote (before and after revascularisation), and control myocardial segments. See fig 2 for key to abbreviations. ${ }^{\star \star *} p<0.0001$.

$(\mathrm{p}=0.0002)$. LVEF remained unchanged after PTCA (41 (12\%) v $43(10 \%), \mathrm{p}=0.16)$ and improved after CABG $(24(7 \%) v 33(9 \%), \mathrm{p}=$ $0.0004)$.

MYOCARDIAL BLOOD FLOW AND CORONARY VASODILATOR RESERVE

\section{Controls}

In the normal subjects, blood flow was homogeneously distributed in the different ventricular regions, and the mean baseline myocardial blood flow was $0.99(0.21) \mathrm{ml} / \mathrm{min} / \mathrm{g}$, increasing to $3.1(1.3) \mathrm{ml} / \mathrm{min} / \mathrm{g}$ after dipyridamole, giving a coronary vasodilator reserve of 3.2 (1.5). The rate-pressure product was 8037 (1798). After correction for the rate-pressure product, myocardial blood flow was 1.03 $(0.29) \mathrm{ml} / \mathrm{min} / \mathrm{g}$ and coronary vasodilator reserve was 3.2 (1.6).

\section{Patients}

In the patients the rate-pressure product before revascularisation was 9337 (2250) and after revascularisation was 8443 (1749) ( $\mathrm{p}=0.1 v$ controls and pre-revascularisation). In the hibernating segments the baseline myocardial blood flow was unchanged after revascularisation while there was a significant increase in the post-dipyridamole myocardial perfusion and the coronary vasodilator reserve (table 1, fig 1). In the remote regions there were no changes in baseline and post-dipyridamole myocardial blood flow and coronary vasodilator reserve after revascularisation (table 2). 
COMPARISON OF THE THREE GROUPS

The baseline myocardial blood flow values before and after revascularisation in hibernating, remote, and control segments were not significantly different (fig 2).

To identify hibernating segments with abnormally low baseline myocardial blood flow before revascularisation, a cut-off of $0.45 \mathrm{ml} /$ $\mathrm{min} / \mathrm{g}$ which corresponds to the mean myocardial blood flow of controls (corrected for RPP) minus 2 SD, was used. Only 10/163 (6\%) hibernating segments had myocardial blood flow lower than this threshold before revascularisation (fig 3).

All pre and post-revascularisation perfusion parameters for hibernating, remote, and control segments are compared in figs 4 and 5.

\section{Discussion}

The main finding of this study is that revascularisation of hibernating myocardium is associated with an increase in coronary vasodilator reserve, while the baseline myocardial blood flow remains unchanged. Furthermore, our data show that in more than $90 \%$ of hibernating segments the myocardial blood flow before revascularisation is within normal range while the coronary vasodilator reserve is significantly lower than in remote regions (in the same patients) and the myocardium of healthy subjects (controls). Previous studies have also demonstrated that the myocardial blood flow of hibernating segments is similar to that of remote regions or healthy control subjects. ${ }^{511} 1516$ Furthermore, Vanovershelde and colleagues demonstrated in a small and selected group of patients that the prerevascularisation coronary vasodilator reserve is reduced in dysfunctional myocardium proved to be hibernating by postrevascularisation contractile improvement. ${ }^{5}$ Our work confirms and expands these findings by providing post-revascularisation data on myocardial blood flow and coronary vasodilator reserve.

Our findings suggest that chronic postischaemic left ventricular dysfunction is not necessarily caused by a chronic reduction in resting blood flow (hibernation in its classical definition), but may be related to a reduction in coronary vasodilator reserve. Reduced coronary vasodilator reserve may result in stress induced left ventricular dysfunction. There is evidence both in animals and humans that this left ventricular dysfunction persists in the postischaemic period despite absence of irreversible damage and in the presence of normal or near normal myocardial blood, and has been termed myocardial stunning. ${ }^{17}{ }^{18}$ In these cases, chronic ventricular dysfunction may be the result of repetitive episodes of imbalance between the supply and demand for oxygen caused by either a temporary increase in myocardial demands (that is, exercise inducible ischaemia) or by oscillations in the blood supply (that is, unstable coronary plaques). Repeated episodes of myocardial ischaemia, occurring during daily life activity, have been documented in patients with chronic stable coronary artery disease. ${ }^{19}$ These authors dem- onstrated ischaemic events to be common and often asymptomatic (73\% of cases) lasting between one and 90 minutes. Persistent left ventricular dysfunction following exercise has also been detected in patients with coronary artery disease. ${ }^{20}$ Furthermore, a cumulative adverse effect on left ventricular dysfunction of repetitive episodes of ischaemia has been demonstrated in animal models of progressive coronary stenosis ${ }^{21} 22$ and confirmed in patients with coronary artery disease. ${ }^{23} 24$ Repetitive episodes of exercise induced ischaemia, followed by stunning, may thus result in a cumulative effect on post-ischaemic myocardial dysfunction, leading to a chronic state of dysfunction.

It could be hypothesised that improvement of contractile function in hibernating myocardium following coronary revascularisation is caused by the improvement in coronary vasodilator reserve, with a reduction of the "repetitive ischaemic events". This may trigger cellular mechanisms to "revert" the ultrastructural and metabolic adaptations resulting from repetitive stunning, thus resulting in an improvement in contractility.

We have used $\mathrm{H}_{2}{ }^{15} \mathrm{O}$ for the measurement of myocardial blood flow; this tracer-unlike ${ }^{13} \mathrm{NH}_{3}$-is not taken up by scar tissue, thus avoiding the dilution effect of scar. ${ }^{4}$

In our study, both the coronary vasodilator reserve of left ventricle segments subtended by an angiographically normal coronary vessel and with normal contractile function (remote regions), and that of hibernating segments after revascularisation, was significantly lower than the coronary vasodilator reserve of controls. These findings are consistent with previous studies demonstrating an abnormal coronary vasodilator reserve in patients with coronary artery disease, even in regions subtended by normal epicardial coronary vessels. ${ }^{12}{ }^{25} \mathrm{Al}-$ though the mechanism underlying this phenomenon remains unknown, others have explained it as a manifestation of microvascular endothelial dysfunction which affects patients with coronary artery disease. ${ }^{26}$

\section{STUDY LIMITATIONS}

In this study we assessed myocardial blood flow, coronary vasodilator reserve, and regional left ventricular function at a single postoperative time point. Serial temporal studies of these parameters could have provided some insight into the time course of improvement in coronary vasodilator reserve and left ventricular function. Although our study demonstrates that hibernating myocardium is associated with a reduced coronary vasodilator reserve, because of the resolution limitations of the current PET scanners we were unable to measure epicardial and endocardial myocardial blood flow and coronary vasodilator reserve, and thus ascertain the implications of these data for the pathophysiology of hibernation. The choice of $0.45 \mathrm{ml} / / \mathrm{min} / \mathrm{g}$ as a lower limit of normal myocardial blood flow is arbitrary and reflects the difficulty of defining normal myocardial blood flow. Studies have demon- 
strated the significant heterogeneity of myocardial blood flow in normal subjects. ${ }^{27} 28$

1 Diamond G, Forrester J, deLuz P, et al. Post-extrasystolic potentiation of ischaemic cardiomyopathy by atrial stimulation. Am Heart $\mathcal{F} 1978 ; 95: 204-9$.

2 Rahimtoola SH. The hibernating myocardium. Am Heart $\mathcal{f}$ 1989;117:211-21.

3 Uren N, Melin J, De Bruyne B, et al. Relation between myocardial blood flow and the severity of coronary artery stenosis. N Engl F Med 1994;330:1782-8.

4 Camici PG, Wijns W, Borgers M, et al. Pathophysiological mechanisms of chronic reversible left ventricular dysfunction due to coronary artery disease (hibernating myocardium). Circulation 1997;96:3205-14

5 Vanoverschelde JJ, Wijns W, Depre' C, et al. Mechanism of chronic regional postischaemic dysfunction in humans. New insights from the study of noninfarcted collateraldependent myocardium. Circulation 1993;87:1513-23.

6 Pagano D, Townend J, Parums D, et al. Hibernating myocardium: morphological correlates of inotropic stimumyocardium: morphological correlates of inotrop

7 Zaret BL, Wackers FJ. Nuclear cardiology (second of two Zaret BL, Wackers FJ. Nuclear cardiology
parts). N Engl f Med 1993;329:855-63.

8 Bland M, Altman D. Statistical methods for assessing agreement between two methods of clinical measurements. Lanment between two

9 Segar D, Brown S, Sawada S, et al. Dobutamine stress echocardiography: correlation with coronary lesion severity as determined by quantitative angiography. $\mathcal{F} \mathrm{Am} \mathrm{Coll} \mathrm{Car}$ diol 1992;19:1197-202.

10 Spinks T, Jones T, Gilardi M, et al. Physical performance of the latest generation of commercially available positron scanner. IEEE Trans Nucl Sci 1988;NS-35:721-5.

11 Marinho N, Keogh B, Costa D, et al. Pathophysiology of chronic left ventricular dysfunction. New insights from the glucose utilisation. Circulation 1996;93:737-44.

12 Uren N, Marraccini P, Gistri R, et al. Altered coronary vasodilatory reserve and metabolism in myocardium vabtended by normal arteries in patients with coronary subtended by normal arteries in patients with
artery reserve. $\mathcal{F} \mathrm{Am}$ Coll Cardiol 1993;22:650-8

13 Czenin J, Muller P, Chan S, et al. Influence of age and haemodynamics on myocardial blood flow and and haemodynamics on myocardial

14 Lorenzoni R, Pagano D, Boyd H, et al. A report system for PET assessment of myocardial viability. Nucl Med Commun 1999;20:895-9.

15 Gerber B, Vanovershelde J-L, Bol A, et al. Myocardial blood flow, glucose uptake, and recruitment of inotropic reserve in chronic left ventricular ischemic dysfunction. Implications for the pathophysiology of chronic myocardial hibernation. Circulation 1996;94:651-9.
16 Grandin C, Wijns W, Melin J, et al. Delineation of myocardial viability with PET. $₹$ Nucl Med 1995;36:1543-52.

17 Heyndrickx G, Millard R, McRitchie R, et al. Regional myocardial function and electrophysiological alterations after brief coronary artery occlusion in conscious dogs. $7 \mathrm{Clin}$ Invest 1975;56:978-85.

18 Ambrosio G, Betocchi S, Pace L, et al. Prolonged impairement of regional contractile function after resolution of exercise-induced angina. Evidence of myocardial stuning in patients with coronary artery disease. Circulation 1996;94:2455-64.

19 Deanfield J, Selwin A, Chierchia S, et al. Myocardial ischaemia during daily life in patients with stable angina: its relation to symptoms and heart rate changes. Lancet 1983;ii: $753-8$.

20 Robertson W, Feigenbaum H, Armstrong W, et al. Exercise echocardiography: a clinically practical addition in the evaluation of coronary artery disease. $\mathcal{F} \mathrm{Am}$ Coll Cardiol 1983;2:1085-91.

21 Shen Y, Vatner S. Mechanism of impaired myocardial function during progressive coronary stenosis in conscious pigs. 88 .

22 Homans D, Laxson D, Sublett E, et al. Cumulative deterioration of myocardial function after repeated episodes of exercise-induced ischaemia. Am $\mathcal{f}$ Physiol 1989;256: H1462-71.

23 Schneider R, Weintraub W, Lloyd W, et al. Rate of left ventricular functional recovery by radionuclide angiography after exercise in coronary artery disease. Am 7 Cardiol 1986;57:927-32.

24 Rinaldi C, Massani N, Linka A, et al. Effect of repetitive episodes of exercise inducible ischaemia on left ventricular function in patients with chronic stable angina: evidence for cumulative stunning or ischaemic preconditioning? Heart. 1999;81:404-11.

25 Sambuceti G, Parodi O, Marcassa C, et al. Alteration in regulation of myocardial blood flow in one-vessel coronary artery disease determined by positron emission tomography. Am f Cardiol 1993;72:538-43.

26 Zeiher A, Drexler H, Wollschlager $\mathrm{H}$, et al. Modulation of coronary vasomotor tone in humans: prograssive endothelial dysfunction with different early stages of coronary atherosclerosis. Circulation 1991;83:391-401.

27 Hoffman J. Heterogeneity of myocardial blood flow. Basic Res Cardiol 1995;90:103-11.

28 Chareonthaitawee P, Kaufmann P, Rimoldi O, et al. Variability of regional myocardial blood flow measured by positron emission tomography in 153 volunteers. Eur Heart f 1999;20 (suppl):P3595. 